\title{
Prevention of Catheter-Related Blood Stream Infection: Back to Basics?
}

\author{
Jill R. Cherry-Bukowiec, Krassimir Denchev, ${ }^{2}$ Sharon Dickinson, ${ }^{3}$ Carol E. Chenoweth, ${ }^{4}$ Christy Zalewski, \\ Craig Meldrum, ${ }^{6}$ Kristen C. Sihler, Melissa E. Brunsvold, Thomas J. Papadimos, \\ Pauline K. Park, and Lena M. Napolitano ${ }^{1}$
}

\begin{abstract}
Background: Central venous catheter (CVC)-related infections are a substantial problem in the intensive care unit (ICU). Our infection control team initiated the routine use of antiseptic-coated (chlorhexidine-silver sulfadiazine; Chx-SS) CVCs in our adult ICUs to reduce catheter-associated (CA) and catheter-related (CR) blood stream infection (BSI) as we implemented other educational and best practice standardization strategies. Prior randomized studies documented that the use of Chx-SS catheters reduces microbial colonization of the catheter compared with an uncoated standard (Std) CVC but does not reduce CR-BSI. We therefore implemented the routine use of uncoated Std CVCs in our surgical ICU (SICU) and examined the impact of this change. Hypothesis: The use of uncoated Std CVCs does not increase CR-BSI rate in an SICU.

Methods: Prospective evaluation of universal use of uncoated Std CVCs, implemented November 2007 in the SICU. The incidences of CA-BSI and CR-BSI were compared during November 2006-October 2007 (universal use of Chx-SS CVCs) and November 2007-October 2008 (universal use of Std CVCs) by $t$-test. The definitions of the U.S. Centers for Disease Control and Prevention were used for CA-BSI and CR-BSI. Patient data were collected via a dedicated Acute Physiology and Chronic Health Evaluation (APACHE) III coordinator for the SICU.

Results: Annual use of CVCs increased significantly in the last six years, from 3,543 (2001) to 5,799 (2006) total days. The APACHE III scores on day 1 increased from a mean of 54.4 in 2004 to 55.6 in 2008 ( $p=0.0010 ; 95 \%$ confidence interval [CI] 1.29-5.13). The mean age of the patients was unchanged over this period, ranging from 58.2 to 59.6 years. The Chx-SS catheters were implemented in the SICU in 2002. Data regarding the specific incidence of CR-BSI were collected beginning at the end of 2005, with mandatory catheter tip cultures when CVCs were removed. Little difference was identified in the incidence of BSI between the interval with universal Chx-SS use and that with Std CVC use. (Total BSI 0.7 vs. 0.8 per 1,000 catheter days; CA-BSI 0.5 vs. 0.8 per 1,000 catheter days; CR-BSI 0.2 vs. 0 per 1,000 catheter days.) No difference was seen in the causative pathogens of CABSI or CR-BSI.

Conclusion: Eliminating the universal use of Chx-SS-coated CVCs in an SICU with a low background incidence of CR-BSIs did not result in an increase in the rate of CR-BSIs. This study documents the greater importance of adherence to standardization of the processes of care related to CVC placement than of coated CVC use in the reduction of CR-BSI.
\end{abstract}

C ENTRAL VENOUS CATHETERS (CVCs) can be life-saving for those who require central access for medications, fluids, and nutrition, but their use is not without risk. In particular, catheter-related (CR) infections are a significant source of morbidity and death in hospitalized patients [1]. The U.S. Centers for Disease Control and Prevention (CDC) estimates

Departments of ${ }^{1}$ Acute Care Surgery and ${ }^{2}$ Anesthesiology, University of Michigan, Ann Arbor, Michigan.

${ }^{3}$ Surgical Intensive Care Unit, University of Michigan.

Departments of ${ }^{4}$ Internal Medicine, ${ }^{5}$ Infection Control, and ${ }^{6}$ Clinical Affairs, University of Michigan.

${ }^{7}$ Department of Anesthesiology, The Ohio State University, Columbus, Ohio. 
an annual occurrence of more than 250,000 CR-BSIs in the United States alone [2]. In addition to the lives lost as a result of CR blood stream infection (BSI), the estimated financial impact of even one BSI can be substantial [3-6]. Because of this, CR-BSIs have been deemed preventable and targeted for eradication by influential medical bodies, including the CDC [2], the Society for Healthcare Epidemiology of America (SHEA) [6], the Infectious Diseases Society of America (IDSA)[7, 8], the 100,000 Lives Campaign, and the 5 Million Lives Campaign from the Institute for Healthcare Improvement [9].

The CDC Guidelines for the Prevention of Intravascular Catheter-Related Infections recommend preventive strategies with strong supportive evidence, including: (1) Education and training of healthcare providers; (2) use of full barrier precautions during CVC insertion; (3) use of a $2 \%$ chlorhexidine preparation for skin antisepsis; (4) no routine replacement of CVCs; and (5) use of an antiseptic/antibiotic-impregnated CVC in adults whose catheter is expected to remain in place longer than five days if, after implementing a comprehensive strategy to reduce the rate of CR-BSI, the infection rate remains above the goal set by the individual institution on the basis of benchmark rates and local factors. All of these preventive strategies are employed by critical care practitioners in an attempt to achieve zero CR-BSIs in intensive care units (ICUs) $[2,10]$.

Antimicrobial-impregnated (minocycline/rifampin) and antiseptic-coated (chlorhexidine-silver sulfadiazine; Chx-SS) CVCs have been studied extensively. Two meta-analyses of randomized trials have established that antimicrobial/ antiseptic-coated CVCs reduce the incidence of microbial colonization and CR-BSI, but the methodologic quality of the studies generally was poor, putting the conclusions in doubt [11]. The minocycline/rifampin CVCs were associated with CR-BSI reduction, but Chx-SS CVCs were not. Furthermore, these studies were not done in settings where infectionprevention bundles of care were established as routine practice. The investigators concluded that coated CVCs may be useful if the incidence of CR-BSI is above institutional goals despite full implementation of infection prevention interventions [12].

However, few studies address the efficacy of antisepticcoated CVCs in ICUs with low CR-BSI rates. Schuerer et al. evaluated whether the use of Chx-SS CVCs would decrease the CR-BSI rate in a surgical ICU (SICU) with an already low rate. They found no statistically significant decrease in CR-BSI rates from before implementation of the Chx-SS CVCs (3.3 per 1,000 catheter days) to after implementation ( 2.1 per 1,000 catheter days; $\mathrm{p}=0.16)[13]$.

The routine use of Chx-SS CVCs was initiated in our SICU as we implemented other educational and standardization strategies to reduce CR-BSI. Once we achieved a low CR-BSI rate, we began using uncoated Std CVCs instead of Chx-SS CVCs and examined the impact of this unit-wide practice change.

\section{Patients and Methods}

\section{Setting}

The University of Michigan 20-bed SICU admits all noncardiothoracic critically ill surgical patients. The SICU operates as a collaborative model in which a dedicated unit team works closely with the admitting surgical team to facilitate communication and coordinate all aspects of care. To minimize duplication and optimize efficiency, only the SICU team enters orders. Physician staffing includes an attending surgical or anesthesiology intensivist and an in-house resident team that consists of a surgical or anesthesiology critical care fellow and resident staff assigned exclusively to the SICU.

\section{Patients}

All patients admitted to the SICU were evaluated for BSIs. Only patients identified as having a BSI who underwent placement of a CVC by the SICU team in the internal jugular, subclavian, or femoral position were included in the analysis.

\section{Design}

We used an interrupted time-series (quasi-experimental) design to examine the outcomes of interest. In 2000, our infection control team initiated the routine use of antisepticcoated triple-lumen CVCs (Chx-SS, first generation with external coating [ARROWguard Blue ${ }^{\circledR}$ ], transitioned to secondgeneration coated internally and externally [ARROWguard Blue PLUS ${ }^{\circledR}$; Arrow International, Inc, Reading PA]) in our adult ICUs as we implemented other educational and standardization strategies aimed at CR-BSI reduction (hand hygiene and antisepsis, dedicated catheter cart use, standardized placement, chlorhexidine skin preparation, maximum sterile barriers) (Fig. 1). Placement, use, and care for CVCs were standardized by hospital policy (see below). Empowerment of any member of the patient care team to stop line placement in the event of contamination or failure of protocol adherence was enforced. Education and twice-daily evaluation of CVC removal was performed during SICU team rounds starting in 2005.

The rate of catheter-associated (CA)-BSI decreased steadily until 2006, during which our rate was 0.9 per 1,000 CVC days and the rate of CR-BSI was 0 per 1,000 CVC days. In November 2007, the SICU team implemented use of standard non-coated triple-lumen CVCs (same manufacturer) and tracked our CA-BSI and CR-BSI rates over the following year. All preventive efforts previously implemented regarding CABSI prevention were continued. Although we did not track compliance with hospital infection control policies, staff education and routine monitoring of hand washing were continued throughout the study period.

\section{Standardization of CVC placement and care}

Placement and maintenance of CVCs is standardized by the University Hospitals and Health Centers Infection Control Committee Policy: VI-58 Central Lines: Temporary Central and Arterial Vascular Access Catheters, Long-Term Central Catheters: Tunneled and Ports, and Peripherally Placed Central Catheters (PICC) [14]. The hospital policy for Use and Maintenance of CVC Catheters was followed, which includes washing hands before and after replacing, accessing, or repairing the catheter, and before and after palpating or dressing the catheter site; and use of clean gloves to remove old dressings and either clean or sterile gloves when placing a fresh, sterile dressing; or using an aseptic "no-touch" technique to apply a sterile dressing to the catheter site. The insertion site is evaluated every $24 \mathrm{~h}$, with every dressing change, and when the patient complains of discomfort at the 


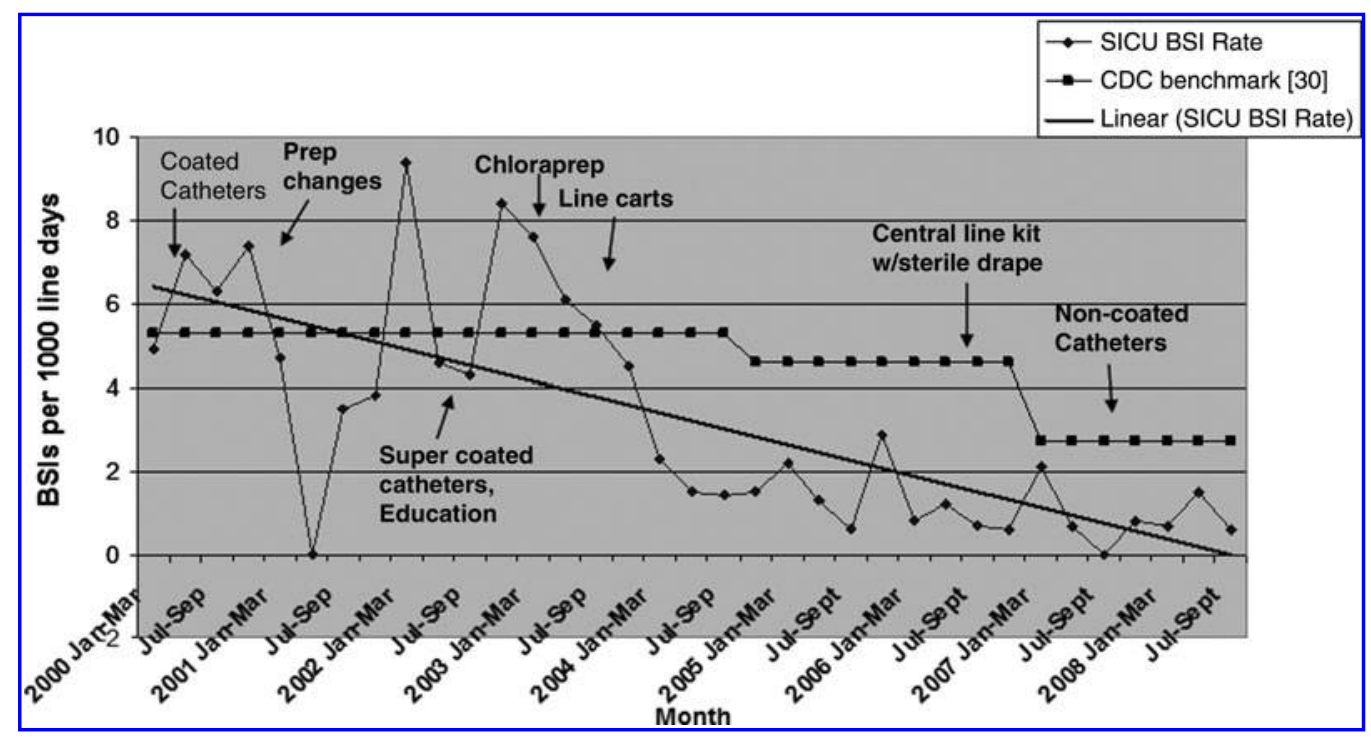

FIG. 1. Surgical intensive care unit blood-stream infection rate, 2000-2008.

site. The catheter site was cleansed during dressing changes with a $2 \%$ chlorhexidine-70\% isopropyl alcohol-based preparation (70\% isopropyl alcohol or iodophor solution if there was a patient allergy). Catheter sites were kept clean with a sterile, dry, gauze dressing taped in a manner to resist moisture; alternatively, a highly permeable transparent dressing was employed.

Dressing changes also are regulated by hospital policy. Gauze dressings or any dressing constructed with gauze or other opaque materials were changed at least every three days or whenever they become wet, loose, or soiled or when inspection of the site was necessary. Highly permeable transparent dressings were changed at least every seven days and as necessary.

Catheter manipulations were kept to a minimum; when necessary, they were performed using strict aseptic technique. A needleless access system with a pierced injection port and blunt cannula or locking blunt cannula was used to maintain a closed system. Access ports were changed every seven days. A Luer-lok-type attachment was used for extension tubing. All stopcocks were fitted with a pierced injection needleless port at the time of set-up. Access points were disinfected with alcohol just before entry. Blood draws through the CVCs were kept to a minimum; when required, blood specimens were obtained using aseptic technique and clean gloves and the tubing, stopcock, IV port, and the access port attached to the stopcock was flushed clean of any remaining blood after the specimen was removed. The use of stopcocks was kept to a minimum, and a fresh, sterile cap was used after each entry. Stopcocks were flushed clean after blood drawing.

The SICU also adheres to University policies for changing the intravenous tubing and attached components (secondary tubing, extension tubing, stopcocks, locking blunt cannulae, pierced injection ports/caps used on stopcocks, Tconnectors). These were changed according to the following guidelines: Tubing for fluids, medications, hemodynamic pressure monitoring, and parenteral nutrition without lipids was changed every $96 \mathrm{~h}$; tubing for lipids and continuous blood products was changed at least every $24 \mathrm{~h}$; and tubing and components used for propofol administration were changed at least every $12 \mathrm{~h}$.
The SICU policy is not to remove or replace CVCs inserted outside the unit routinely if they were placed with aseptic technique. If CVCs are placed in an emergency situation or under non-sterile conditions, they are removed immediately. It is our policy never to replace CVCs over a guidewire unless it is impossible to place a new CVC elsewhere. The internal jugular site is preferred for CVC placement in the SICU, with real-time ultrasound guidance to avoid technical complications and to avoid subclavian vein stenosis in patients with advanced renal disease.

\section{Data collection}

Differentiation between CA-BSI and CR-BSI was first made in 2005, mandating catheter tip cultures and peripheral blood cultures in all SICU patients when CVCs were removed. A CA-BSI was defined as a positive blood culture in a patient with a CVC in place and no other apparent source of infection; i.e., the CVC is assumed to be the cause of the BSI. A CR-BSI was defined as a positive blood culture in a patient with a CVC in place, isolation of the same organism from a semiquantitative or quantitative culture of a catheter segment and from the blood of a patient with symptoms of BSI, and no other apparent source of infection; i.e., the catheter is confirmed to be the cause of the BSI. Cultures from CVCs were performed by the central microbiology laboratory using the roll plate method, in which the cut end of the CVC catheter is rolled across an agar plate and the numbers of colony-forming units are counted after an incubation period [15]. Blood stream infections were monitored by the University infection control team, and the data were evaluated monthly by the faculty, staff, and administration at the SICU quality assurance meeting.

\section{Statistics}

We used an unpaired $t$-test to compare the Acute Physiology and Chronic Health Evaluation (APACHE) III score, Acute Physiology Score (APS), and age differences in the SCIU from the year before intervention with those of the postintervention period. A $2 \times 2$ contingency table was analyzed 
Table 1. Patient Demographics and Critical Care Characteristics

\begin{tabular}{lrr}
\hline & Pre-intervention & Post-intervention \\
\hline No. of patients in surgical ICU & 1,305 & 1,227 \\
Mean patient age (years) (range) & $56.8(13-103)$ & $56.7(13-94)$ \\
Percent of patients aged $\geq 65$ years & 32.1 & 33.3 \\
Mean APACHE III, ICU day 1 (range) & $52.4(4-188)$ & $55.6(7-214)$ \\
Mean Acute Physiology Score, ICU day 1 (range) & $42.4(4-188)$ & $45.3(7-198)$ \\
No. of patients on mechanical ventilation, ICU day 1 & 548 & 528 \\
Admission therapy level (\%) & & 811 \\
$\quad$ Active treatment & $851(63.6)$ \\
$\quad$ High-risk monitor & $119(8.8)$ & $(12.2)$ \\
$\quad$ Low risk monitor & $377(27.8)$ & 301 \\
Mean length of stay (days) & $(23.6)$ \\
$\quad$ ICU & 4.6 & 4.9 \\
$\quad$ Hospital & 13.9 & 18.2 \\
Mean ventilator days & 3.9 & 4.2 \\
\hline
\end{tabular}

$\mathrm{APACHE}=$ Acute Physiology and Chronic Health Evaluation score; ICU = intensive care unit.

using the $\chi^{2}$ test with Yates correction to compare total BSI, CA-BSI, and CR-BSI rates from the pre-intervention period with those of the post-intervention period.

\section{Results}

Patients

The number of SICU admissions annually ranged from 1,340 to 1,491 . The services admitting patients to the SICU included Transplant, Non-Trauma Emergency, Trauma, General Surgery, Surgical Oncology, Urology, Obstetrics/ Gynecology, Orthopedics, Otolaryngology, Vascular Surgery, Interventional Radiology, Thoracic Surgery, and Surgical Critical Care. Disease acuity, assessed by the admission APACHE III score [16], increased from 52.5 in the preintervention period (historical 58.2 in 2004) to 55.6 in the postintervention period $(\mathrm{p}=0.0010 ; 95 \%$ confidence interval $[\mathrm{CI}]$ 1.29-5.13). Similarly, the mean APS [16] increased from 42.3 to 45.3 ( $\mathrm{p}=0.0009 ; 95 \%$ CI 1.22-4.74) during the same time. The mean patient age remained the same at 57 years. There were no differences in any other demographics or critical care characteristics of the SICU patients during the time periods examined (Table 1).

\section{Catheter-associated and catheter-related blood stream infections}

The total number of CVC days during the pre-intervention period (November 2006-October 2007) when Chx-SS-coated triple-lumen CVCs were used exclusively was 5,580. The mean number of catheter-days per patient was 5.79 in the preintervention period and 5.87 in the post-intervention period. The total number of CA-BSIs was three, a rate of 0.5 per 1,000 catheter-days. The total number of CR-BSIs during the preintervention period was one, a CR-BSI rate of 0.2 per 1,000 catheter-days (Table 2).

The total number of CVC days during the post-intervention period (November 2007-October 2008) when Std non-coated triple-lumen CVCs were in use was 5,244. The total number of CA-BSIs was four, a rate of 0.8 per 1,000 catheter-days. The total number of CR-BSIs during the time period was zero (Tables 2 and 3).

\section{Causative pathogens}

No difference in the causative microbial pathogens for CABSI or CR-BSI was noted between the pre-intervention and post-intervention periods (Table 3 ). Note that most of these

Table 2. Catheter-Associated and Catheter-Related Blood Stream Infection Rates in the Surgical INTENSIVE CARE Unit Over Time

\begin{tabular}{|c|c|c|c|c|c|c|c|c|}
\hline Month/year & $\begin{array}{c}\text { Catheter } \\
\text { type }\end{array}$ & $\begin{array}{c}\text { Catheter } \\
\text { days }\end{array}$ & $\begin{array}{c}\text { Total } \\
\text { BSI }\end{array}$ & $\begin{array}{c}\text { Total } \\
\text { BSI rate }\end{array}$ & $C A-B S I$ & $\begin{array}{c}C A-B S I \\
\text { rate }\end{array}$ & $C R-B S I$ & $\begin{array}{c}C R-B S I \\
\text { rate }\end{array}$ \\
\hline \multicolumn{9}{|l|}{ Total } \\
\hline 2001 & Chx-SS & 3,543 & 11 & 3.1 & $\mathrm{~N} / \mathrm{A}$ & $\mathrm{N} / \mathrm{A}$ & $\mathrm{N} / \mathrm{A}$ & $\mathrm{N} / \mathrm{A}$ \\
\hline 2002 & Chx-SS & 3,824 & 26 & 6.8 & $\mathrm{~N} / \mathrm{A}$ & $\mathrm{N} / \mathrm{A}$ & $\mathrm{N} / \mathrm{A}$ & $\mathrm{N} / \mathrm{A}$ \\
\hline 2003 & Chx-SS & 3,713 & 22 & 5.9 & $\mathrm{~N} / \mathrm{A}$ & $\mathrm{N} / \mathrm{A}$ & $\mathrm{N} / \mathrm{A}$ & $\mathrm{N} / \mathrm{A}$ \\
\hline 2004 & Chx-SS & 5,385 & 9 & 1.6 & $\mathrm{~N} / \mathrm{A}$ & $\mathrm{N} / \mathrm{A}$ & $\mathrm{N} / \mathrm{A}$ & $\mathrm{N} / \mathrm{A}$ \\
\hline 2005 & Chx-SS & 5,645 & 10 & 1.8 & $\mathrm{~N} / \mathrm{A}$ & $\mathrm{N} / \mathrm{A}$ & $\mathrm{N} / \mathrm{A}$ & $\mathrm{N} / \mathrm{A}$ \\
\hline 2006 & Chx-SS & 5,799 & 5 & 0.9 & 5 & 0.9 & 0 & 0 \\
\hline 2007 & Chx-SS & 5,395 & 5 & 0.9 & 4 & 0.7 & 1 & 0.2 \\
\hline 11/06-10/07 (pre-intervention) & Chx-SS & 5,580 & 4 & 0.7 & 3 & 0.5 & 1 & 0.2 \\
\hline $11 / 07-10 / 08$ (post-intervention) & Std & 5,244 & 4 & 0.8 & 4 & 0.8 & 0 & 0 \\
\hline
\end{tabular}

$\mathrm{BSI}=$ blood stream infection; $\mathrm{CA}=$ catheter-associated; $\mathrm{CR}=$ catheter-related; $\mathrm{Chx}-\mathrm{SS}=$ chlorhexidine-silver sulfadiazine central venous catheter; $\mathrm{N} / \mathrm{A}=$ not available; $\mathrm{Std}=$ standard catheter.

${ }^{a}$ Rate $=$ central venous catheter infections per 1,000 catheter-days. 
Table 3. Organisms Cultured from Catheter-Associated and Catheter-Related Blood Stream Infections, Central Venous Catheter (CVC) Position, and Days from Catheter Insertion to Infection

\begin{tabular}{lllr}
\hline CA-BSI or CR-BSI & \multicolumn{1}{c}{ Organism } & \multicolumn{1}{c}{ Vein used } & Days from CVC insertion to BSI \\
\hline $\begin{array}{l}\text { Pre-intervention (11/06-10/07) } \\
\text { CA-BSI }\end{array}$ & Bacteroides spp. & Right internal jugular & 6 \\
CA-BSI & VSE & Left internal jugular & 20 \\
CA-BSI & Candida parapsilosis & Right femoral & 15 \\
CR-BSI & VRE & Right femoral & 10 \\
Post-intervention (11/07-10/08) & & & \\
CA-BSI & VRE & Right internal jugular & 4 \\
CA-BSI & VSE & Left femoral & 26 \\
CA-BSI & Serratia spp. & Left femoral & 4 \\
CA-BSI & Enterobacter spp. & Left internal jugular & 8 \\
Post-evaluation (11/08-4/09) & & & \\
CA-BSI & VRE & Left subclavian & 6 \\
\hline
\end{tabular}

$\mathrm{CA}=$ catheter-associated; $\mathrm{CR}=$ catheter- related; $\mathrm{BSI}=$ blood stream infection; $\mathrm{CVC}=$ central venous line; VSE $=$ vancomycin-sensitive Enterococcus; VRE = vancomycin-resistant Enterococcus.

CA-BSIs were not confirmed as CR-BSIs, and the microbial pathogens identified probably were not the causative pathogens in the CR infections.

\section{Relation of catheter location to infection}

Catheter-associated BSIs were commonly identified in CVCs positioned in the internal jugular or femoral vein (Table 3). Subclavian CVC placement is not often performed in our SICU because the use of real-time ultrasound guidance is employed regularly for safety concerns.

\section{Discussion}

It has been estimated that CVCs account for approximately $90 \%$ of all blood stream infections $[17,18]$, and more than five million CVCs are inserted per year in the U.S. [19,20]. Antimicrobial/antiseptic-coated and -impregnated catheters have been designed in an attempt to reduce CR-BSI. Multiple metaanalyses have concluded that coated CVCs are associated with reduction in the rates of BSI, but these studies were performed in the era when CR-BSI rates were high [20-24].

A number of studies have documented that implementation of an education program and standardization of CVC placement in addition to full application of the CDC guidelines for prevention of CA-BSI is associated with a significant reduction in CA-BSI, without the use of antimicrobial/ antiseptic-coated CVCs [25-27]. An important singleinstitution study documented that the "recipe" for zero CRBSI included: (1) Standardized education of staff; (2) standardized placement of CVC; (3) having a CVC cart with all necessary supplies; (4) daily inquiry regarding discontinuation of CVC; (5) catheter-insertion checklist for assurance of aseptic technique; and (6) empowering the team to halt the procedure if aseptic technique rules are violated [28]. A similar intervention was implemented state-wide in Michigan (108 ICUs; 375,757 catheter days) without the use of antimicrobial/antiseptic-coated CVCs, and a significant reduction in mean CA-BSI, from 7.7 to $1.4 / 1,000$ catheter-days, was documented, representing an overall $66 \%$ reduction [29].

After years of an aggressive education program, implementation of the CDC guidelines, and use of Chx-SScoated CVCs, we reduced the incidence of CA-BSI in the SICU to very low rates. We next examined whether Chx-SS CVCs were an essential component of our CR-BSI infection prevention efforts. By replacing the Chx-SS CVCs with noncoated Std CVCs, we introduced a single change in our CR-BSI infection prevention efforts that did not result in an increase in either CA-BSI or CR-BSI. Our findings are similar to those of Schuerer et al., who demonstrated that use of ChxSS-coated catheters in a SICU with an already low CA-BSI rate failed to reduce the rate further [13].

We continue to use uncoated Std CVCs exclusively in the SICU. Importantly, neither the CA-BSI nor the CR-BSI rate in the SICU changed substantially over the following five months (CABSI total one [0.5 per 1,000 catheter-days]; CR-BSI total zero.)

Catheter-associated BSI has been proposed to be and is accepted as a "never" event; however, this is difficult to achieve. Despite maximum multidisciplinary efforts to reduce CA-BSI in a single ICU as a controlled environment, a persistent zero rate of CA-BSI was not achievable.

The interrupted time-series (quasi-experimental) design used to examine the impact of this intervention (use of uncoated Std CVCs) in the SICU does have several limitations. Most notably, it was conducted in a single SICU in a single tertiary-care institution with all the inherent biases, and is potentially subject to influence by changes in patient populations, ICU practices and policies, and other unrecognized temporal biases. We did not collect data on the CVCs that were not associated with BSI and therefore cannot make any comments on the location, indwelling time, or specific patient characteristics associated with CVCs not linked to BSI. Additionally, in the SICU, CVCs are likely to be placed in a specific subset of critically ill patients. The types of patients and the reason for CVC placement were not tracked during this study.

Despite these limitations, the findings of this analysis support the conclusion that aggressive education programs and adherence to best-practice guidelines are powerful tools in the prevention of CA-BSI and CR-BSI. In ICUs where exceptionally low CA-BSI rates already have been achieved, the universal use of Chx-SS-coated catheters does not yield any additional reduction. Given the cost of $\$ 50.00$ to $\$ 100.00$ more for a Chx-SS than an Std catheter, use of Std CVCs in ICUs with a low CRBSI rate carries potential cost savings. 


\section{Conclusion}

Elimination of the universal use of Chx-SS-coated CVCs in an SICU with a low number of CR-BSIs did not increase the rate of such infections. This study documents the greater importance of adherence to standardization of the processes of care related to CVC placement and care than of catheter design in reducing CR-BSI.

\section{Author Disclosure Statement}

No conflicting financial interests exist.

\section{References}

1. Raad I, Hanna H, Maki D. Intravascular catheter-related infections: Advances in diagnosis, prevention, and management. Lancet Infect Dis 2007;10:645-657.

2. O'Grady NP, Alexander M, Dellinger EP, et al. Guidelines for the prevention of intravascular catheter-related infections. Centers for Disease Control and Prevention. MMWR Recomm Rep 2002;51:1-29.

3. Laupland KB, Lee H, Gregson DB, Manns BJ. Cost of intensive care unit-acquired bloodstream infections. I Hosp Infect 2006;63:124-132.

4. Warren DK, Quadir WW, Hollenbeak CS, et al. Attributable cost of catheter-associated bloodstream infections among intensive care patients in a nonteaching hospital. Crit Care Med 2006;34:2084-2089.

5. Kilgore ML, Ghosh K, Beavers CM, et al. The costs of nosocomial infections. Med Care 2008;46:101-104.

6. LeDell K, Muto CA, Jarvis WR, Farr BM. SHEA guideline for preventing nosocomial transmission of multidrug-resistant strains of Staphylococcus aureus and Enterococcus. Infect Control Hosp Epidemiol 2003;24:639-641.

7. Mermel LA, Allon M, Bouza E, et al. Clinical practice guidelines for the diagnosis and management of intravascular catheter-related infection: 2009 Update by the Infectious Diseases Society of America. Clin Infect Dis 2009;49:1-45.

8. Marschall J, Mermel Leonard A, Classen D, et al. Strategies to prevent central line associated bloodstream infections in acute care hospitals. Infection Control Hosp Epidemiol 2008; 29:S22-S30.

9. Protecting 5 Million Lives from Harm. IHI.org [updated 2009; cited April 27, 2009]. Available at www.ihi.org/IHI/ Programs/Campaign/.

10. Raad II. Commentary: Zero tolerance for catheter-related bloodstream infections: The unnegotiable objective. Infect Control Hosp Epidemiol 2008;29:951-953.

11. Hockenhull JC, Dwan KM, Smith GW, et al. The clinical effectiveness of central venous catheters treated with anti-infective agents in preventing catheter-related bloodstream infections: A systematic review. Crit Care Med 2009;37:702-712.

12. Casey AL, Mermel LA, Nightingale P, Elliott TS. Antimicrobial central venous catheters in adults: A systematic review and meta-analysis. Lancet Infect Dis 2008;8:763-776.

13. Schuerer DJ, Zack JE, Thomas J, Borecki IB, et al. Effect of chlorhexidine/silver sulfadiazine-impregnated central venous catheters in an intensive care unit with a low blood stream infection rate after implementation of an educational program: A before-after trial. Surg Infect 2007;8:445-454.

14. Marschall J, Mermel LA, Classen D, et al. Strategies to prevent central line-associated bloodstream infections in acute care hospitals. Infect Control Hosp Epidemiol 2008;29(Suppl 1):S22-S30.

15. Hockenhull JC, Dwan K, Boland A, et al. The clinical effectiveness and cost-effectiveness of central venous catheters treated with anti-infective agents in preventing bloodstream infections: A systematic review and economic evaluation. Health Technol Assess 2008;12:iii-iv, xi-xii, 1-154.

16. Zimmerman JE, Wagner DP, Draper EA, et al. Evaluation of Acute Physiology and Chronic Health Evaluation III predictions of hospital mortality in an independent database. Crit Care Med 1998;26:1317-1326.

17. Le Gall JR, Lemeshow S, Saulnier F. A new simplified Acute Physiology Score (SAPS II) based on a European/North American multicenter study. JAMA 1993;270:2957-2963.

18. Mer M, Duse AG, Galpin JS, Richards GA. Central venous catheterization: A prospective, randomized, double-blind study. Clin Appl Thromb Hemost 2009;15:19-26.

19. Kalfon P, de Vaumas C, Samba D, et al. Comparison of silverimpregnated with standard multi-lumen central venous catheters in critically ill patients. Crit Care Med 2007;35:1032-1039.

20. Raad I, Reitzel R, Jiang Y, et al. Anti-adherence activity and antimicrobial durability of anti-infective-coated catheters against multidrug-resistant bacteria. J Antimicrob Chemother 2008;62:746-750.

21. Crnich CJ, Maki DG. The promise of novel technology for the prevention of intravascular device-related bloodstream infection I: Pathogenesis and short-term devices. Clin Infect Dis 2002;34:1232-1242.

22. Marin MG, Lee JC, Skurnick JH. Prevention of nosocomial bloodstream infections: Effectiveness of antimicrobial-impregnated and heparin-bonded central venous catheters. Crit Care Med 2000;28:3332-3338.

23. Ramritu P, Halton K, Collignon P, et al. A systematic review comparing the relative effectiveness of antimicrobial-coated catheters in intensive care units. Am J Infect Control 2008;36:104-117.

24. Veenstra DL, Saint S, Saha S, et al. Efficacy of antisepticimpregnated central venous catheters in preventing catheter-related bloodstream infection: A meta-analysis. JAMA 1999;281:261-267.

25. Coopersmith CM, Rebmann TL, Zack JE, et al. Effect of an education program on decreasing catheter-related bloodstream infections in the surgical intensive care unit. Crit Care Med 2002;30:59-64.

26. Coopersmith CM, Zack JE, Ward MR, et al. The impact of bedside behavior on catheter-related bacteremia in the intensive care unit. Arch Surg 2004;139:131-136.

27. Warren DK, Zack JE, Mayfield JL, et al. The effect of an education program on the incidence of central venous catheter-associated bloodstream infection in a medical ICU. Chest 2004;126:1612-1618.

28. Berenholtz SM, Pronovost PJ, Lipsett PA, et al. Eliminating catheter-related bloodstream infections in the intensive care unit. Crit Care Med 2004;32:2014-2020.

29. Pronovost P, Needham D, Berenholtz S, et al. An intervention to decrease catheter-related bloodstream infections in the ICU. N Engl J Med 2006;355:2725-2732.

30. Edwards JR, Peterson KD, Andrus ML, et al. National Healthcare Safety Network (NHSN) Report: Data summary for 2006 through 2007, issued November 2008. Am J Infect Control 2008;36:609-626.

Address correspondence to: Dr. Jill R. Cherry-Bukowiec University of Michigan University Hospital 1C421 1500 E. Medical Center Dr., SPC 5033 Ann Arbor, MI 48109

E-mail: jillcher@med.umich.edu 